\title{
Extração de Contornos de Telhados de Edificações através da Combinação de Dados Lidar e Imagens Aéreas
}

\author{
Extraction of Building Roof Boundaries through the integration of LiDAR \\ Data and Aerial Images \\ Gilmar Renan Kisaki Oliveira ${ }^{1}$ \\ Mauricio Galo ${ }^{2}$
}

Recebido em fevereiro de 2017.

Aprovado em outubro de 2018.

\begin{abstract}
RESUMO
Este artigo contempla o desenvolvimento de um método que combina os dados obtidos por sistema de varredura a LASER (Light Amplification by Stimulated Emission of Radiation) aerotransportado e imagens aéreas a fim de extrair os contornos de telhados de edificações. Os pontos de contorno das edificações são extraídas dos dados LiDAR e são projetados em duas imagens que formam um modelo estereoscópico. A estas imagens, cujos parâmetros de orientação são conhecidos, são aplicados o algoritmo de detecção de bordas de Canny com o objetivo de identificar as bordas de edificações no espaço imagem. Com os contornos dos telhados de edificações, provenientes dos dados LiDAR projetados nas imagens de bordas é realizado o refinamento das bordas a partir da busca dos pontos de bordas de edificações nas imagens. Com base nos pixels identificados como bordas de edificações e com o propósito de obter os modelos matemáticos que representem os contornos, é aplicado o ajuste de retas 2D pelo Método dos Mínimos Quadrados (MMQ) integrado à filtragem de pontos espúrios por meio do Teste Tau. Para avaliar o método proposto e implementado foram utilizados dados LiDAR com densidade média de 6,7 pontos $/ \mathrm{m}^{2}$ e imagens aéreas digitais com GSD de $8 \mathrm{~cm}$. Os resultados obtidos na avaliação dos experimentos mostraram que o método proposto conseguiu extrair os contornos dos telhados, com melhores resultados para edificações isoladas que não possuíam projeção de sombras ou objetos sobre elas, atingindo valores da ordem de 0,97 GSD e 1,80 GSD, para o REMQ em planimetria e altimetria, respectivamente.
\end{abstract}

\footnotetext{
${ }_{1}^{1}$ Universidade Estadual Paulista (Unesp), Faculdade de Ciências e Tecnologia Programa de Pós-Graduação em Ciências Cartográficas (PPGCC); renan.kisaki@gmail.com

${ }^{2}$ Universidade Estadual Paulista (Unesp), Faculdade de Ciências e Tecnologia.

Programa de Pós-Graduação em Ciências Cartográficas (PPGCC) -

Departamento de Cartografia. Presidente Prudente - SP; mauricio.galo@fct.unesp.br
} 
PALAVRAS-CHAVE: Dados LiDAR. ajuste pelo MMQ. biblioteca LAStools. teste Tau. deteç̧ão de bordas por Canny.

\begin{abstract}
This article proposes a methodology to extract building boundary through the integration of LiDAR data and aerial imagery where the image orientation parameters are known. Building are first extracted from LiDAR data, then building

boundaries are determined and projected onto the stereo pair of aerial images. These aerial images are results from the application of Canny edge detector in order to identify building boundaries from images. Since the 3D building boundaries determined from LiDAR data are projected onto the Canny images, a search mechanism is performed to find the building boundaries based on the edge points near the projected points. A 2D line adjustment by Least Squares Method (LSM) is performed, followed by outlier detection based on Tau statistical test, for generating a geometric shape to represent the buildings through the building edge pixels identified. In order to evaluate the proposed approach, LiDAR data with approximate density of $6,7 \mathrm{pts} / \mathrm{m}^{2}$ and digital aerial images with GSD around $8 \mathrm{~cm}$ were used. The results showed that the proposed method enabled to extract building roof boundaries with best results for isolated buildings without objects or shadow's projection on them with the root mean square error (RMSE) around 0,97 GSD and 1,80 GSD in planimetry and altimetry, respectively.
\end{abstract}

KEYWORDS: LiDAR data. Least Square Method. LAStools. Tau statistical test. Canny edge detection.

$$
* * *
$$

\title{
Introdução
}

Em aplicações cartográficas, a extração de edificações cumpre um papel fundamental no que diz respeito à aquisição e atualização de dados espaciais de um Sistema de Informações Geográficas (SIG), uma vez que estas informações podem ser utilizadas como ferramenta de apoio na tomada de decisões em setores estratégicos de planejamento. Neste contexto, buscando encontrar soluções viáveis para a extração de edificações, muitos estudos vêm sendo realizados por pesquisadores das áreas de Fotogrametria e Sensoriamento Remoto por meio de diferentes fontes de dados, tais como imagens aéreas ou de satélite; dados obtidos por sistemas de varredura a LASER (Light Amplification by Stimulated Emission of Radiation); ou, até mesmo, a integração de ambos. 
A integração entre dados provenientes de Fotogrametria e de sistemas baseados na tecnologia LiDAR (Light Detection And Ranging) torna-se atrativa, tendo em vista as características complementares que estas duas fontes de dados possuem. A questão de como combinar estes diferentes dados de uma maneira ideal, para que os seus pontos fracos possam ser compensados de forma eficaz é objeto de decorrentes estudos (YONG e HUAYI, 2008).

Os dados fotogramétricos são caracterizados pela elevada redundância por meio da observação de objetos em múltiplas imagens (HABIB et al., 2004). No entanto, os sistemas LiDAR, que emergiram como uma revolucionária tecnologia de coleta de dados da superfície física da Terra, apresentam certas vantagens em relação aos métodos fotogramétricos convencionais. Uma de suas principais características é a geração de uma densa nuvem de pontos com coordenadas tridimensionais da superfície terrestre de forma rápida e precisa, essencial na modelagem de superfícies, por exemplo.

De acordo com Habib et al. (2004), as imagens e os dados LiDAR possuem características distintas que tornam uma ou outra preferencial para certas aplicações. A integração de dados LiDAR e imagens fornece soluções mais precisas, combinando a altimetria do sistema LiDAR e a planimetria de imagens aéreas (AWRANGJEB et al., 2010). A natureza posicional dos dados LiDAR é ideal para o mapeamento de superfícies homogêneas. Por outro lado, carecem de qualidade na representação de objetos que possuem linhas de descontinuidade (HABIB, 2004), ao contrário das imagens que fornecem bordas de feições bem definidas. Entretanto, é importante destacar que, cada vez mais, sistemas LiDAR têm sido aprimorados, possibilitando a obtenção de maior densidade de pontos, fazendo com que objetos que possuem linhas de descontinuidade possam ser representados de forma mais fidedigna.

A extração de contornos de edificações de forma acurada não é uma tarefa trivial, devido às distintas formas geométricas das edificações e à 
complexidade do ambiente circundante que existe em regiões urbanas resultando em diversas limitações no processo de extração.

Uma vez reconhecida a natureza complementar dos dados LiDAR e imagens, assim como a complexidade no processo de extração de edificações de forma semiautomática ou automática, diversos estudos têm sido realizados nos últimos anos neste âmbito (KABOLIZADE et al., 2010; KHOSHELHAM et al., 2010; LIU et al., 2011; MARCATO et al., 2014; ZHAI, 2015; GILANI et al., 2016; KARSLI et al., 2016).

Apesar dos êxitos atingidos, nota-se o grande interesse no estudo de novas metodologias para a extração de edificações, uma vez que ainda há diversas limitações envolvidas no processo.

Neste contexto, este artigo apresenta o desenvolvimento de uma metodologia a fim de extrair contornos de telhados de edificações por meio da integração dos dados provenientes do sistema LiDAR e imagens aéreas digitais de uma mesma área urbana.

\section{Método}

As principais etapas do método proposto são: preparação dos dados de entrada; extração de edificações nos dados LiDAR; extração de contornos de edificações nos dados LiDAR; e refinamento dos contornos de edificações integrando dados LiDAR e imagens.

1.1 Preparação dos dados de entrada

Os dados de entrada para a aplicação do método proposto são recortes de regiões correspondentes sobre os dados LiDAR e as imagens ópticas 
disponíveis. Uma vez que os experimentos visam avaliar o método proposto optou-se por trabalhar com recortes das imagens, onde estão presentes edificações de diferentes complexidades.

$\mathrm{Na}$ etapa da análise de qualidade do método proposto, deve-se determinar a posição 3D dos cantos das edificações. Com este propósito são utilizadas duas imagens que formam par estereoscópico, onde estão localizados os recortes em que foram realizados os experimentos. Sendo assim, os procedimentos realizados em uma imagem são análogos aos realizados em outra.

Considerando os recortes das imagens das áreas de estudo, é aplicado o detector de bordas de Canny a fim de detectar os contornos dos telhados de edificações. O recorte das imagens e aplicação do detector de bordas de Canny são realizados por meio de macros que são executadas no aplicativo ImageJ, visando a automatização desses procedimentos. É importante ressaltar que os parâmetros requeridos para o algoritmo de detecção de bordas de Canny (desvio-padrão $\sigma$, e limiares alto $\tau_{1}$ e baixo $\tau_{2}$ ) foram determinados empiricamente por meio de testes feitos em recortes das imagens.

\subsection{Extração de edificações nos dados LiDAR}

A etapa de extração de edificações nos dados LiDAR visa a extração das edificações na área de estudo, para posterior identificação dos pontos que delimitam suas bordas. O procedimento para o cumprimento desta etapa é realizado por meio de funções da biblioteca LAStools incorporadas ao código implementado em Linguagem C no compilador Code::Blocks.

Os dados LiDAR correspondentes às áreas selecionadas são classificadas, por meio da função lasground, em duas categorias: pontos de terreno e não terreno. Esta função utiliza o método de filtragem progressiva para classificar os pontos, como pode ser visto em Dal Poz (2013). Para a sua 
aplicação quatro parâmetros podem ser modificados, de acordo com as características da região:

- Step: O valor desse parâmetro (unidade: $\mathrm{m}$ ) está relacionado com a quantidade de objetos altos na região. Quanto maior for este parâmetro, menor a probabilidade de um objeto alto como edifício ou vegetação ser considerado, por exemplo, como ponto de terreno;

- Spike: Remove picos acima e abaixo do valor do parâmetro adotado (unidade: $\mathrm{m}$ );

- Stddev: Considera certo desvio padrão para os pontos de terreno em áreas planas (unidade: $\mathrm{cm}$ );

- Offset: Classifica como pontos de terreno aqueles que possuírem altura até o valor adotado (unidade: $\mathrm{m}$ ).

Como dados de saída têm-se as coordenadas 3D de cada ponto (X, Y, Z) e sua classificação, onde pontos classificados como terreno recebem o atributo 2 e aqueles classificados como não terreno, o atributo 1.

$\mathrm{Na}$ sequência, é aplicada a função lasheight a fim de calcular a altura de cada ponto acima do terreno. Assume-se que os pontos de terreno já foram classificados e, desta forma, eles podem ser identificados e utilizados para a geração de um MDT por meio de uma rede irregular de triângulos (TIN Triangular Irregular Network). Sendo assim, é calculada a altura de cada ponto classificado como não terreno em relação a essa estrutura TIN. Essa função permite que as alturas de todos os pontos acima do terreno sejam normalizadas por meio da opção 'replace_z', ou seja, a altura dos pontos classificados como terreno recebem o valor 0 e os pontos de não terreno, a mesma altura em relação ao TIN, em uma dada posição (X,Y). Desta forma, como resultado, tem-se o MDSn da região.

Outra possibilidade é calcular a altura de cada ponto e eliminar aqueles que possuem altura acima ou abaixo que um limiar adotado por meio das opções '-drop_below' ou 'drop_above'. 
Com o MDSn da região, é possível realizar a classificação dos pontos referentes aos objetos altos em edificação ou vegetação por meio da função lasclassify que analisa a altura e o padrão de distribuição dos pontos de uma certa vizinhança que podem ter características plana (edificação) ou irregular (vegetação). Para a utilização dessa função, assume-se que os pontos de terreno já tenham sido classificados pela função lasground e a altura de cada ponto acima do terreno tenha sido calculada por meio da função lasheight. Essa função possui três parâmetros que podem ser alterados conforme as características dos dados:

- Planar: Verifica se a vizinhança é plana considerando o limiar de planaridade. Os pontos abaixo do limiar adotado são classificados como edificação (unidade: $\mathrm{cm}$ );

- Rugged: Verifica se a vizinhança é irregular. Os pontos acima do limiar adotado são classificados como vegetação (unidade: $\mathrm{cm}$ );

- Ground_offset: Limiar relacionado à altura mínima que um ponto deve ter para ser classificado como edificação ou vegetação (unidade: $\mathrm{m})$.

Como dados de saída têm-se arquivos . ${ }^{*}$ txt com as coordenadas $3 \mathrm{D}$ de cada ponto $(\mathrm{X}, \mathrm{Y}, \mathrm{Z})$ e sua classificação. A rotulação usada na classificação segue o que foi estabelecido pela ASPRS (2013). Deste modo, os pontos classificados como edificação recebem o atributo 6 e aqueles classificados como vegetação, o atributo 3. Uma vez classificado o MDSn, é possível separar os pontos correspondentes às edificações dos demais.

A nuvem de pontos referentes às edificações é regularizada, resultando, desta forma, numa grade regular de pontos por meio da função lasgrid. Esta função cria uma malha bidimensional permitindo a definição do espaçamento que se deseja entre os pontos.

1.3 Extração de contornos de edificações nos dados LiDAR 
Nesta etapa, as edificações são separadas individualmente e são extraídos os pontos de seu contorno nos dados LiDAR. Como dados de entrada tem-se a grade regular correspondente às edificações. $\mathrm{O}$ procedimento de separação automática das edificações é baseado no algoritmo de crescimento de região por meio do critério de vizinhança e altura implementado por Barbosa e Galo (2015) e a extração dos contornos das edificações realizada por meio da função lasboundary da biblioteca LAStools.

\subsubsection{Separação automática das edificações nos dados LiDAR}

Uma vez que os dados LiDAR são compostos por pontos irregularmente espaçados, diferentemente dos pixels em uma imagem, é feita previamente a interpolação dos dados LiDAR correspondentes às edificações para a geração de uma grade regular como mencionado na Seção 1.2.

O processo da seleção automática das edificações se inicia com a definição de um ponto semente. Para tanto, por meio do canto esquerdo superior da malha regular, o algoritmo percorre as linhas continuamente até que se encontre algum ponto que possua valor de altura diferente de zero, podendo, então, ser definido como ponto semente. O algoritmo compara o ponto semente com seus vizinhos, podendo-se considerar o tipo de vizinhança4 ou vizinhança- 8 .

Com o tipo de vizinhança definido, o algoritmo inicia a busca de pontos com altura diferente de zero e que possua uma diferença de altura em relação ao ponto semente, considerando um dado limiar. Ao final do processo de busca considerando a vizinhança adotada, um novo ponto semente é selecionado e em função dos pontos adicionados como pertencentes à mesma feição, o processo de teste é reiniciado. O algoritmo considera que não haja mais pontos a serem adicionados como pertencentes a uma certa feição quando todos os pontos que já tenham sido adicionados como pertencentes a ela forem testados (BARBOSA e GALO, 2015). 
Como dados de saída têm-se os arquivos no formato .*xyz contendo os pontos de cada uma das edificações isoladas.

1.3.2 Extração dos pontos de contornos das edificações

Esta etapa consiste na identificação dos pontos que envolvem o contorno das edificações realizada por meio da função lasboundary da biblioteca LAStools. Esta função permite gerar o menor polígono que englobe o conjunto de pontos externos a um conjunto de pontos. Com base no parâmetro '-concavity' é possível estabelecer a concavidade do polígono externo. Quanto maior o valor deste parâmetro, menor a quantidade de pontos que envolve o contorno do polígono.

Uma vez extraído todos os pontos que compõe o contorno dos telhados, o próximo passo consiste na separação de cada um dos lados desse contorno. Para tanto, uma possibilidade é determinar os pontos inicial e final de cada lado do telhado pela análise do ângulo de deflexão $\left(\theta_{\mathrm{d}}\right)$ dos vetores formados pelos pontos $3 \mathrm{D}$ dos contornos, tendo em vista que, teoricamente, os cantos da edificação apresentam ângulos de deflexão maiores que os ângulos de deflexão para os pontos das laterais, como ilustra a Figura 1.

Figura 1 - Ângulo de deflexão entre os pontos de contorno. 


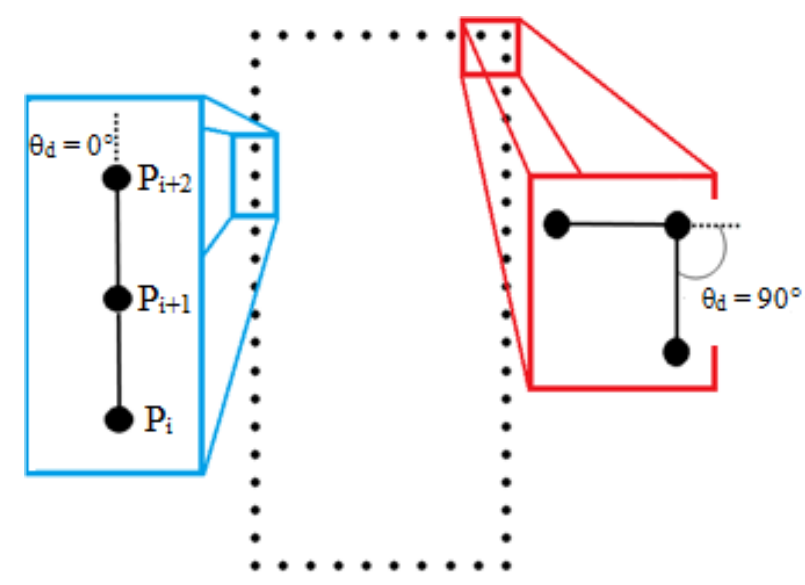

Fonte: Elaborado pelos autores.

Considerando os pontos 3D adjacentes $\mathrm{Pi}, \mathrm{Pi}+1$ e $\mathrm{Pi}+2$ (Figura 1) e (u, v) como os vetores formados por $(\mathrm{Pi}, \mathrm{Pi}+1)$ e $(\mathrm{Pi}+1, \mathrm{Pi}+2)$, respectivamente, o ângulo de deflexão do ponto $\mathrm{Pi}+1$ pode ser calculado por:

$$
\theta_{\mathrm{d}}=\arccos \left(\frac{\mathrm{u} \cdot \mathrm{v}}{\|\mathrm{u}\|\|\mathrm{v}\|}\right)
$$

$\mathrm{Na}$ análise dos ângulos de deflexão é adotado um intervalo considerando dois limiares L1 e L2, onde os valores dos ângulos calculados para cada ponto são analisados e verificados se estão dentro do intervalo estabelecido. Caso estejam, eles são considerados como pontos extremos dos lados da edificação.

Conhecendo o ponto inicial e final de cada lado da edificação, é possível obter os contornos de cada lado individualizados.

Dependendo da amostragem e densidade dos dados LiDAR, bem como do resultado da segmentação por crescimento de região, os pontos de contorno dos telhados das edificações resultantes podem se comportar de maneira irregular e, desta forma, pontos que não são extremos dos lados da edificação podem apresentar ângulos de deflexão dentro do intervalo estabelecido. Para solucionar este problema, uma possibilidade é determinar novamente o ângulo de deflexão, mas agora, somente entre os pontos candidatos a pontos 
extremos das retas que possuíam ângulos dentro do intervalo estabelecido e, considerando um novo limiar, identificar os pontos que apresentam ângulos de deflexão maiores que o limiar adotado.

1.4 Extração de contornos de edificações integrando dados LiDAR e imagens

Nesta subseção é descrita a integração entre os pontos de contorno das edificações obtidos sobre os dados LiDAR e as bordas de Canny detectadas nas imagens para a extração dos contornos de telhados finais. Ao utilizar dados de diferente natureza é essencial garantir que ambos estejam disponibilizados no mesmo referencial. Neste caso, assume-se que os parâmetros de orientação das imagens sejam conhecidos, de modo que seja possível o registro da nuvem de pontos LiDAR com as imagens disponíveis.

Esta etapa envolve três principais fases: projeção do contorno das edificações obtidos nos dados LiDAR sobre as imagens de borda de Canny; busca da borda de Canny das edificações que são mais próximos aos pontos de contorno das edificações obtidos nos dados LiDAR; e o ajuste de retas dos pixels de borda pelo Método dos Mínimos Quadrados (MMQ) a fim de obter uma figura geométrica que represente os lados dos telhados de edificações.

1.4.1 Busca dos contornos de telhados na imagem por meio da integração com os dados LiDAR

Ao analisar dados LiDAR percebe-se que os contornos de telhados são regiões onde as linhas de descontinuidade estão presentes. Devido ao processo de amostragem dos dados LiDAR não é possível garantir que todos os pontos da borda de um telhado, por exemplo, sejam amostrados. Aliado a isso, temse também um erro posicional associado à aquisição dos dados LiDAR. Deste modo, as imagens podem ser utilizadas como uma importante fonte para se 
obter contornos de telhados com maior qualidade, uma vez que possuem qualidade semântica e geométrica com alto grau de detalhamento.

Neste contexto, a ideia deste método é utilizar os contornos dos telhados obtidos pelos dados LiDAR para auxiliar na identificação dos contornos dos telhados nas imagens de bordas de Canny.

Os dados de entrada nesta etapa incluem: os pontos 3D dos contornos das edificações obtidos a partir dos dados LiDAR e as imagens de bordas de Canny.

Uma vez conhecidos os pontos inicial e final de cada lado da edificação nos dados LiDAR, os pontos de cada lado individualmente são projetados nas imagens de bordas, onde é realizada a busca das bordas detectadas por Canny.

A Figura 2 mostra o procedimento realizado para a busca do pixel de borda, detectado por Canny (representado com $\mathrm{ND}=0$, em preto), que seja mais próximo do ponto de borda detectado a partir dos dados LiDAR projetado na imagem (representado na cor cinza).

Figura 2 - Busca do pixel de borda mais próximo do ponto LiDAR projetado na imagem.

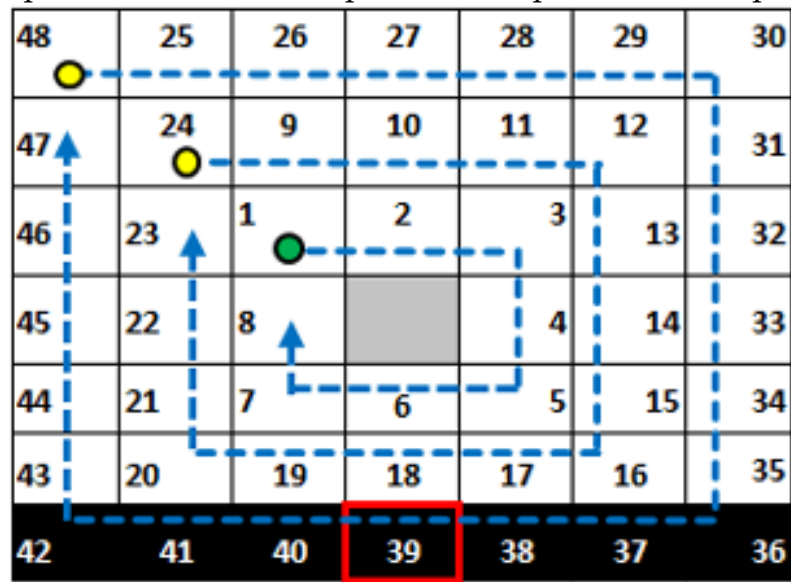

Ponto inicial

O Ponto de continuidade

- Pixel de borda de Canny

Pixel do ponto LiDAR projetado

- Pixel de borda mais próximo

Fonte: Elaborado pelos autores.

Rev. Bras. de Cartografia, vol. 70, n. 4, outubro/dezembro, 2018. pp. 1378 - 1408. 
A busca do pixel de borda na imagem, mais próximo do pixel do ponto LiDAR projetado, se inicia por meio da análise dos rótulos, conforme mostrado na Fig. 2. Caso o ponto LiDAR projetado coincida com um ponto com $\mathrm{ND}=0$, implica que sua posição é coincidente à de um ponto de borda e a busca para este pixel do ponto LiDAR projetado se encerra. Caso contrário, a partir do pixel superior esquerdo ao pixel do ponto LiDAR, o algoritmo percorre a vizinhança ao redor do pixel do ponto LiDAR no sentido horário (pixels 1 até 8) até que se encontre algum pixel que possua $\mathrm{ND}=0$. Caso encontre, a distância entre o pixel projetado (a partir do ponto LiDAR) e o pixel candidato é calculada e comparada com um limiar de distância adotado (d'). Se a distância for menor que o limiar, o pixel candidato é considerado como o pixel de borda mais próximo até então. O procedimento continua até que se complete a primeira volta ao redor do pixel proveniente do ponto LiDAR projetado, onde a cada ponto candidato é calculada a sua distância ao pixel do ponto LiDAR e comparada com a menor distância até aquele momento. Desta forma, caso encontre algum pixel candidato na primeira volta, como resultado terá o pixel de borda mais próximo. Caso contrário, o algoritmo continua a busca a partir do pixel 24 (Figura 2) e realiza o mesmo procedimento até encontrar o pixel candidato mais próximo do pixel do ponto LiDAR, ou até que a distância do pixel do ponto LiDAR aos pixels de busca seja menor que o limiar adotado (d') no início do processo.

Este procedimento é realizado até que todos os pontos 3D (provenientes dos dados LiDAR) referentes ao lado da edificação e que foram projetados na imagem, sejam analisados. Sendo assim, como resultado têm-se as coordenadas $(\mathrm{c}, \mathrm{l})=($ coluna, linha) dos pixels de borda de Canny mais próximos aos pixels dos pontos LiDAR projetados.

Assumindo que o objetivo é modelar as bordas que são retas, adota-se o modelo geométrico de uma reta $2 \mathrm{D}$ como a equação da figura geométrica que represente os lados dos telhados de edificações, que são projetados no plano 
imagem. Deste modo é feito o ajuste pelo MMQ integrado à filtragem de pontos espúrios por meio do teste Tau.

O teste Tau é um dos métodos para a identificação de erros grosseiros (outliers). Ele foi introduzido por Pope (1976) e baseia-se num teste estatístico que emprega a distribuição Tau, na qual é utilizado quando o fator de variância a priori não é conhecido. No caso desse trabalho, é empregado para a identificação e eliminação de pixels de borda que não pertencem às edificações, visando melhorar a estimação dos parâmetros.

De acordo com Pope (1976) e Camargo (2000), o teste é feito a partir da formulação de duas hipóteses: hipótese básica $\left(\mathrm{H}_{0}\right)$, o resíduo da observação $\mathrm{i}$ $\left(\mathrm{v}_{\mathrm{i}}\right)$ estimado no ajustamento tem média nula; e hipótese alternativa $\left(\mathrm{H}_{1}\right)$, o resíduo vi não é nulo, ou seja:

$$
\mathrm{H}_{0}: \mathrm{v}_{\mathrm{i}}=0 \text { contra } \mathrm{H}_{1}: \mathrm{v}_{\mathrm{i}} \neq 0
$$

Para validar uma das hipóteses, deve-se comparar o resíduo padronizado $\left(\mathrm{t}_{\mathrm{i}}\right)$ dado por:

$$
\mathrm{t}_{\mathrm{i}}=\mathrm{v}_{\mathrm{i}} / \sigma \mathrm{v}_{\mathrm{i}}
$$

com $1 \leq \mathrm{i} \leq \mathrm{n}$, onde $\mathrm{n}$ é o número de observações; $\mathrm{v}_{\mathrm{i}}$ é o resíduo e $\sigma \mathrm{v}_{\mathrm{i}}$ é o desviopadrão do i-ésimo resíduo.

A estatística $\mathrm{t}_{\mathrm{i}}$ tem distribuição Tau, com gl graus de liberdade, ou seja, $\mathrm{t}_{\mathrm{i}} \sim \tau_{\mathrm{gl}}$. A hipótese básica não é rejeitada a um determinado nível de significância $\alpha_{0}$, se:

$$
-\tau_{\mathrm{gl}}<t_{\mathrm{i}}<\tau_{\mathrm{gl}}
$$

onde $\tau_{\mathrm{gl}}=\sqrt{\frac{\mathrm{gl} \cdot \mathrm{t}_{\mathrm{gl}-1}^{2}}{\mathrm{gl}-1+\mathrm{t}_{\mathrm{gl} l 1}^{2}}}$. 
Caso contrário, conclui-se que a observação i contém erro. De acordo com Camargo (2000), esse teste permite analisar uma observação por vez e dependendo da magnitude do erro, mais de um resíduo pode exceder os valores críticos, detectando mais de uma observação com erro. Ao final da análise de todas as observações, a que possuir a maior estatística, daquelas que se encontram fora do intervalo crítico, deve ser excluída. O teste é repetido até que todas as observações com erro sejam eliminadas. No caso desse trabalho, tais observações são os possíveis pixels que não pertencem às bordas de edificações detectadas por Canny.

1.5 Determinação dos cantos das edificações no espaço objeto

Após a aplicação do procedimento descrito nas seções anteriores às duas imagens do par estereoscópico têm-se as coordenadas planimétricas dos respectivos cantos das edificações. Uma vez que os parâmetros de orientação exterior das imagens são disponíveis, as coordenadas 3D destes pontos, e, portanto, das edificações no espaço objeto podem ser obtidas a partir da interseç̧ão fotogramétrica via equações de colinearidade, como descrito com detalhes em Oliveira (2016).

\section{Resultados Experimentais}

Para testar e verificar a aplicabilidade do método proposto e implementado foram utilizados dados disponibilizados pela German Society for Photogrammetry, Remote Sensing and Geoinformation (DGPF) no website da International Society for Photogrammetry and Remote Sensing (ISPRS) referente à cidade Vaihingen localizada na Alemanha (CRAMER, 2010). Estes dados correspondem aos dados LiDAR e imagens aéreas digitais, juntamente com seus parâmetros de orientação. Os dados LiDAR possuem densidade média de aproximadamente 6,7 pontos $/ \mathrm{m}^{2}$ e as imagens, 
infravermelha falsa-cor, são pré-corrigidas das distorções e possuem GSD (Ground Sample Distance) de $8 \mathrm{~cm}$.

Um aspecto importante na aplicação deste procedimento é garantir que os dados estão no mesmo referencial e registrados. A nuvem de pontos utilizada neste trabalho foi pós-processada pelo DGPF por meio de um ajuste entre faixas a fim de corrigir os erros sistemáticos no processo de georreferenciamento. Foram utilizadas como primitivas os planos extraídos das imagens obtidas com a câmara Intergraph/ZI DMC. Desta forma, o georreferenciamento dos dados LiDAR é compatível com a orientação exterior das imagens (ROTTENSTEINER et al., 2013).

Considerando os recortes das imagens de três áreas de estudo, foi aplicado o detector de bordas de Canny a fim de detectar os contornos dos telhados de edificações.

Conforme Canny (1986) recomenda, na aplicação do detector de bordas foi considerado o limiar baixo $\tau_{2}$ como um terço do limiar alto $\tau_{1}$. Os valores $\tau_{1}=\mathrm{x}$ e $\tau_{2}=\mathrm{y}$ foram adotados empiricamente, por meio de testes realizados com os dados disponíveis. Deve-se destacar que estes valores podem variar de acordo com a qualidade da imagem (relação sinal ruído - SNR), disposição dos objetos em cena, presença de sombra, dentre outras possíveis influências.

Nas etapas envolvidas na extração de edificações nos dados LiDAR foram utilizadas as funções lasground, lasheight e lasclassify da biblioteca LAStools, onde os parâmetros utilizados na função lasground foram: step $=10 \mathrm{~m} ;$ spike $=1 \mathrm{~m} ;$ stddev $=10 \mathrm{~cm} ;$ e offset $=0,05 \mathrm{~m}$. A Figura 3 mostra os resultados da classificação pela função lasground para as três áreas de estudo. Os pontos de terreno estão representados na cor verde e os pontos de não terreno, tais como vegetação e telhados de edificações, aparecem na cor vermelha.

Fig. 3 - Resultado da classificação em pontos de terreno e não terreno para (a) Área 1, (b) Área 2 e (c) Área 3.

Rev. Bras. de Cartografia, vol. 70, n. 4, outubro/dezembro, 2018. pp. 1378 - 1408. 


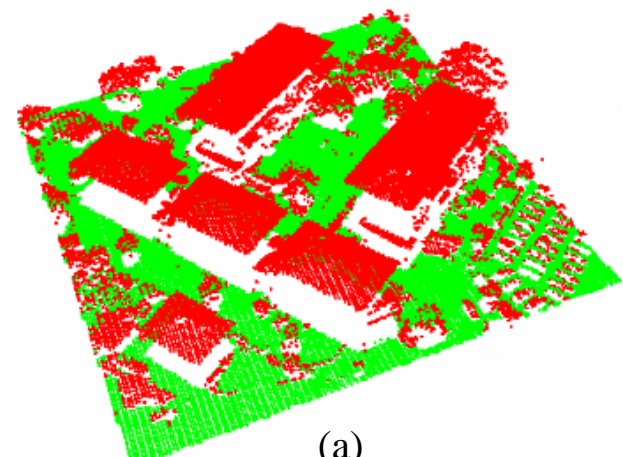

(a)

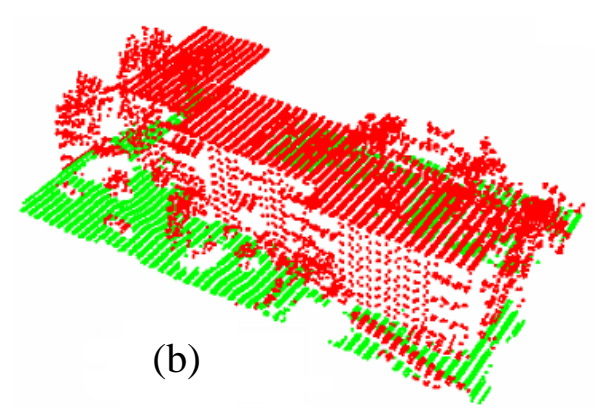

(b)

(c)

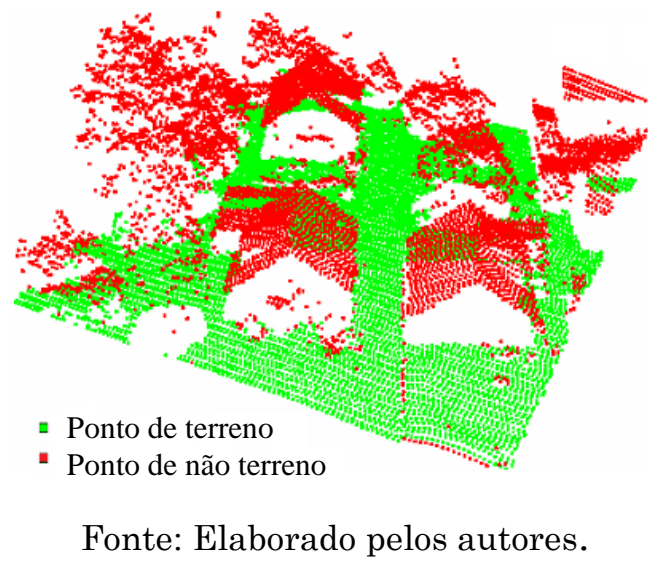

Na sequência, os pontos de terreno foram excluídos e, então, aplicou-se a função lasheight a fim de calcular a altura de cada ponto acima do terreno. Desta forma, foi feita a classificação dos pontos em edificação ou vegetação por meio da função lasclassify. Foram considerados os seguintes parâmetros: planar $=0,1 \mathrm{~m} ;$ rugged $=0,4 \mathrm{~m}$; e ground_offset $=2 \mathrm{~m}$. A Figura 4 mostra os resultados da classificação para as três áreas de estudo. Os pontos de edificação estão representados na cor vermelha, os pontos de vegetação na cor verde e os pontos não classificados em amarelo.

Fig. 4 - Resultado da classificação para (a) Área 1, (b) Área 2 e (c) Área 3. 


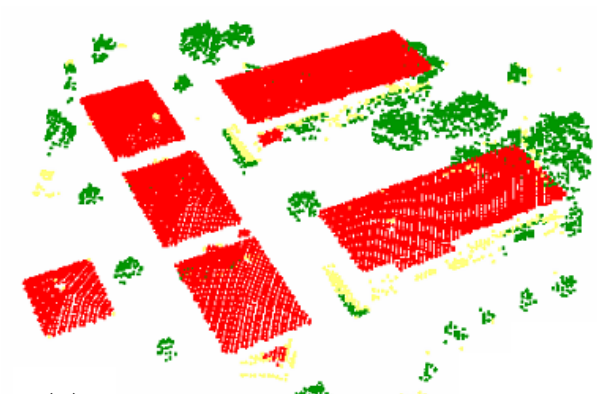

(a)

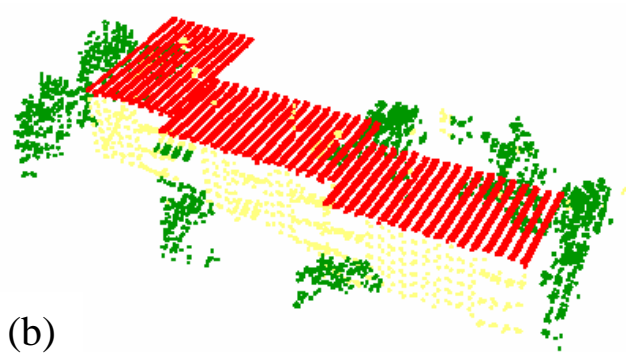

(c)

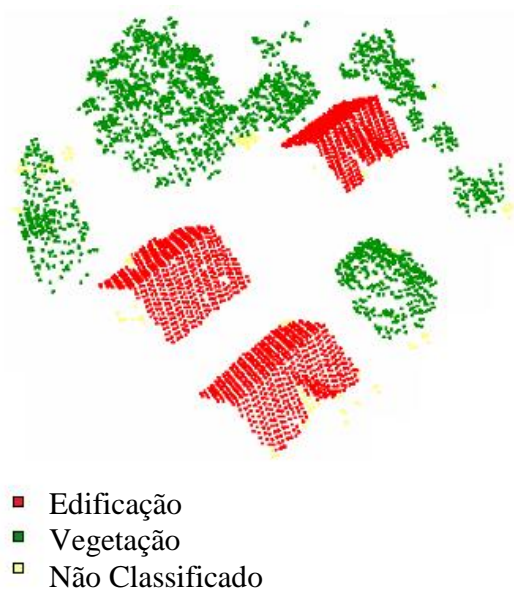

Fonte: Elaborado pelos autores.

No procedimento de separação automática das edificações nos dados LiDAR, baseado no algoritmo de crescimento de região, foram considerados limiares iguais para todas as áreas de estudo. Consideraram-se como edificações as feições que apresentassem um número de pontos igual ou superior a 300 pontos, o que corresponde a considerar edificações que possuem área equivalente a pelo menos $27 \mathrm{~m}^{2}$. Além disso, foi considerado como limiar de desnível o valor de $30 \mathrm{~cm}$ e como critério de vizinhança o padrão de 8 vizinhos.

A Figura 5 mostra o resultado da separação automática das edificações sobre os dados LiDAR baseado no algoritmo de crescimento de região para as áreas de estudo. 
Fig. 5 - Resultado da separação automática das edificações nos dados LiDAR baseado no algoritmo de crescimento de região para (a) Área 1, (b) Área 2 e (c) Área 3.

(a)

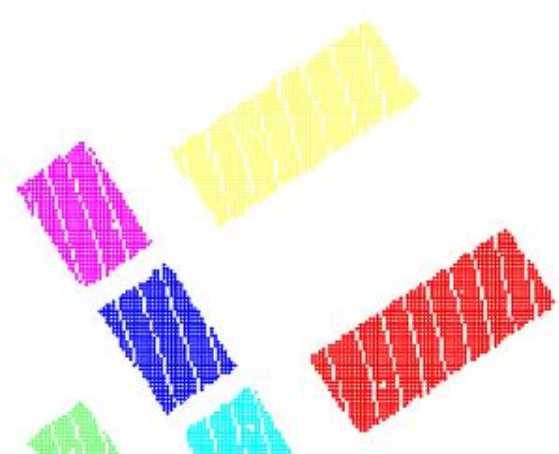

(b)
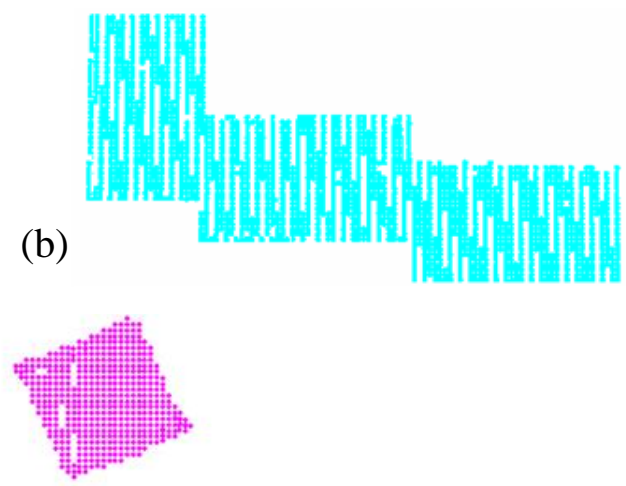

(c)

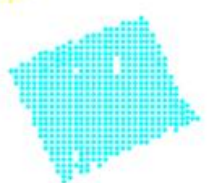

Fonte: Elaborado pelos autores.

Quanto ao resultado referente à Área 2 (Figura 5), o algoritmo não obteve êxito em separar as edificações. Isso pode ser explicado pelo fato de que as edificações são conjugadas e com pequena diferença de altura entre os blocos vizinhos. Desta forma, as edificações foram reconhecidas como uma única feição.

$\mathrm{Na}$ etapa de extração dos pontos de contornos das edificações que, consistiu na identificação dos pontos que envolvem o contorno das edificações realizada por meio da função lasboundary da biblioteca LAStools foi possível estabelecer a não convexidade do polígono externo com base no parâmetro 'concavity'. Para tanto, considerou-se o valor 2 , sendo este valor adotado empiricamente por meio de testes realizados. 
A Figura 6 mostra o resultado da identificação dos pontos que envolvem o contorno das edificações realizada por meio da função lasboundary para as áreas de estudo.

Fig. 6 - Resultado da identificação dos pontos de contorno das edificações por meio da função lasboundary para Área 1 (a), Área 2 (b) e Área 3 (c).

(a)

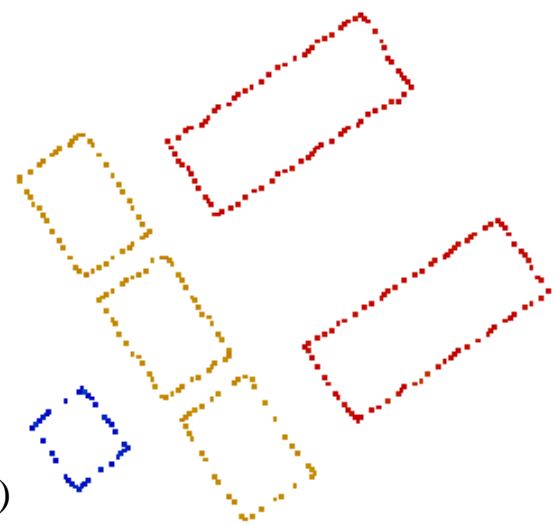

(b)

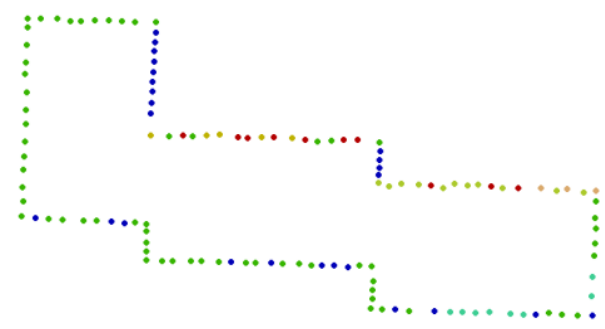

(c)

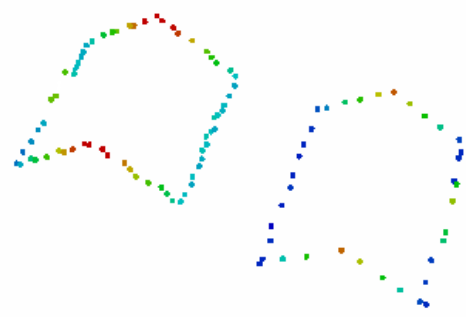

Fonte: Elaborado pelos autores.

Uma vez conhecidos os pontos inicial e final de cada lado da edificação nos dados LiDAR é feita a análise do ângulo de deflexão $\left(\theta_{d}\right)$ dos vetores formados pelos pontos dos contornos como descrito na Seção 2.3.2. Os pontos de cada lado individualmente foram projetados nas imagens de bordas, onde foi realizada a busca das bordas detectadas por Canny mais próximas. $\mathrm{O}$ detector de Canny identifica as bordas de todos os objetos presentes nas cenas, de acordo com os parâmetros adotados. Caso o registro entre os dados LiDAR 
e as imagens não seja de qualidade, o procedimento de busca das bordas de Canny mais próximas aos pontos provenientes dos dados LiDAR, poderá resultar em falsos pontos de bordas referentes às edificações.

Com os pixels de bordas de Canny mais próximos encontrados, para cada feição nas respectivas imagens dos pares, foi feito ajuste de retas $2 \mathrm{D}$ pelo MMQ seguida da aplicação do teste Tau.

Na Figura 7 são apresentados os pontos de borda após o ajuste das retas para as imagens do par das áreas de estudo. 
Fig. 7 - Pontos de bordas de Canny resultantes do processo de ajuste de retas e teste sobre os recortes das imagens aéreas da esquerda (a) e direita (b) para as áreas de estudo.
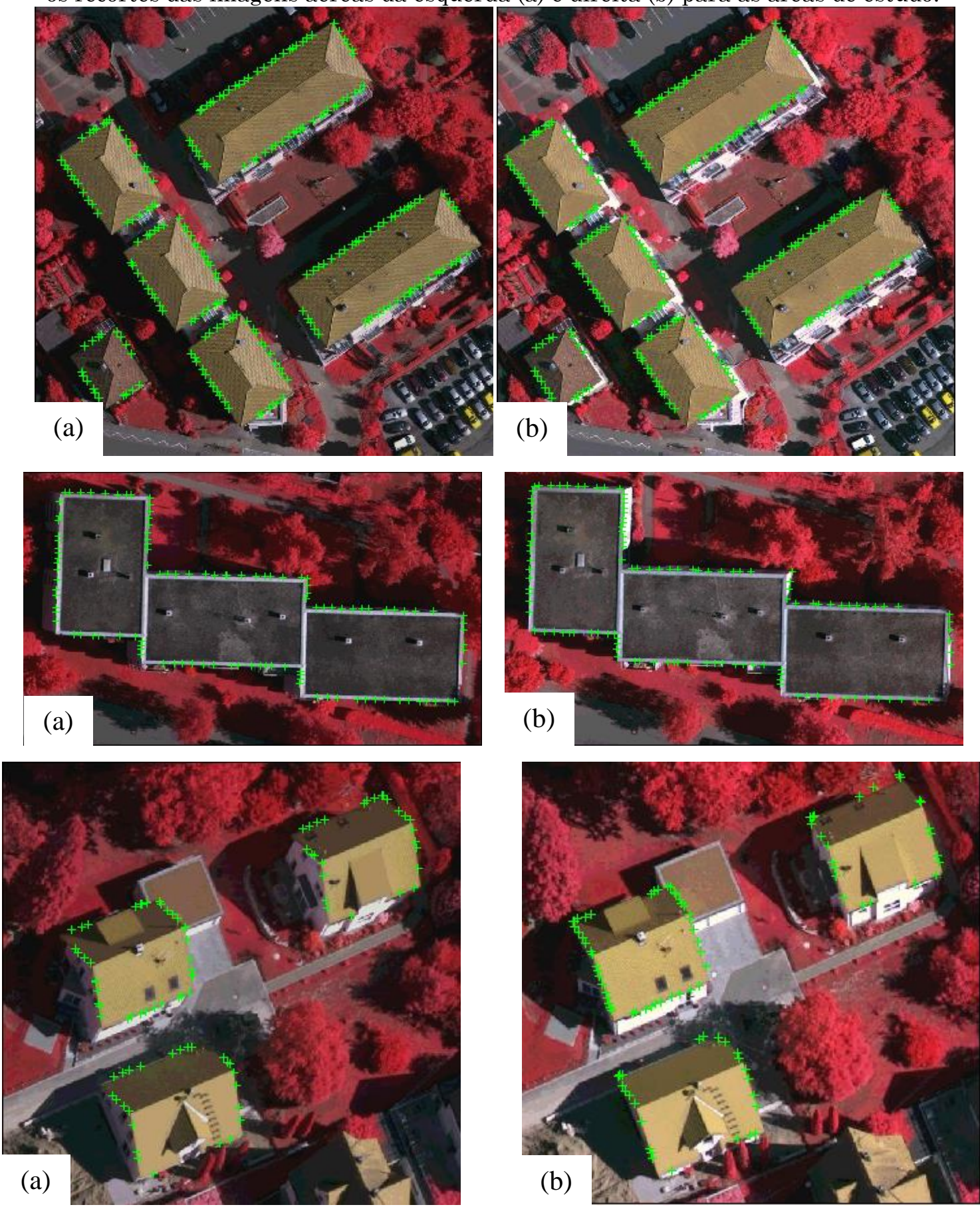

Fonte: Elaborado pelos autores.

Com pode-se notar na Figura 7, a maioria dos pontos de bordas de Canny resultantes do ajuste de retas condiz com os contornos reais das 
edificações. No entanto, pôde-se observar a presença de sombra de árvores projetada numa porção de um telhado da Área 1 (Figura 8a) e da Área 3 (Figura 8c), enquanto na Figura 8b, verificou-se a sombra de uma das edificações da Área 2 projetada na parede de outra.

Fig. 8 - (a) e (c) Sombras de árvores projetadas sobre os telhados; (b) sombra da edificação vizinha projetada sobre a parede de outra edificação.

(a)

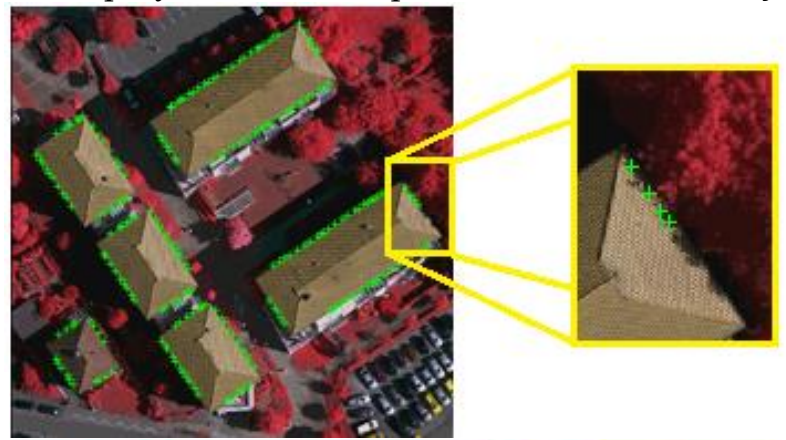

(b)

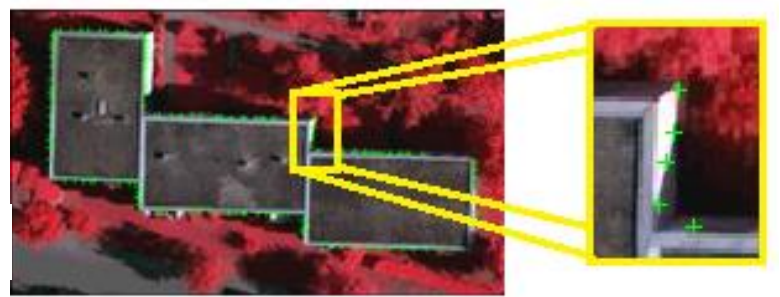

(c)

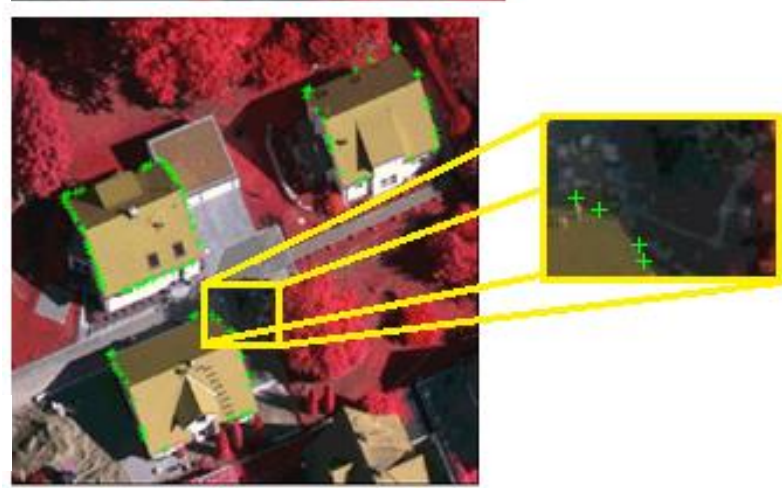

Fonte: Elaborado pelos autores

Em função dos problemas apontados, mostrados em destaque, a borda detectada por Canny não resultou na real borda dos telhados, mas na borda das sombras projetadas. Sendo assim, na busca dos pixels de bordas mais próximos dos pontos LiDAR de contorno projetados, os pixels de borda da sombra foram os mais próximos e, desta forma, considerados pixels de bordas 
de edificações. Esta situação explica o fato dos pixels resultantes do ajuste de retas pertencerem ao contorno das sombras projetadas.

Com os pontos de bordas de Canny resultantes do processo de ajuste de retas pelo MMQ e teste Tau referentes a cada lado dos telhados de edificações, foi possível, com os parâmetros das retas obtidas, realizar a intersecção entre elas a fim de obter os cantos dos telhados. As Figuras 9, 10 e 11 mostram as retas ajustadas referentes aos contornos dos telhados de edificações para cada área de estudo.

Fig. 9 - Cantos e contornos finais obtidos sobre a imagem da esquerda (a) e imagem da direita (b) para Área 1.
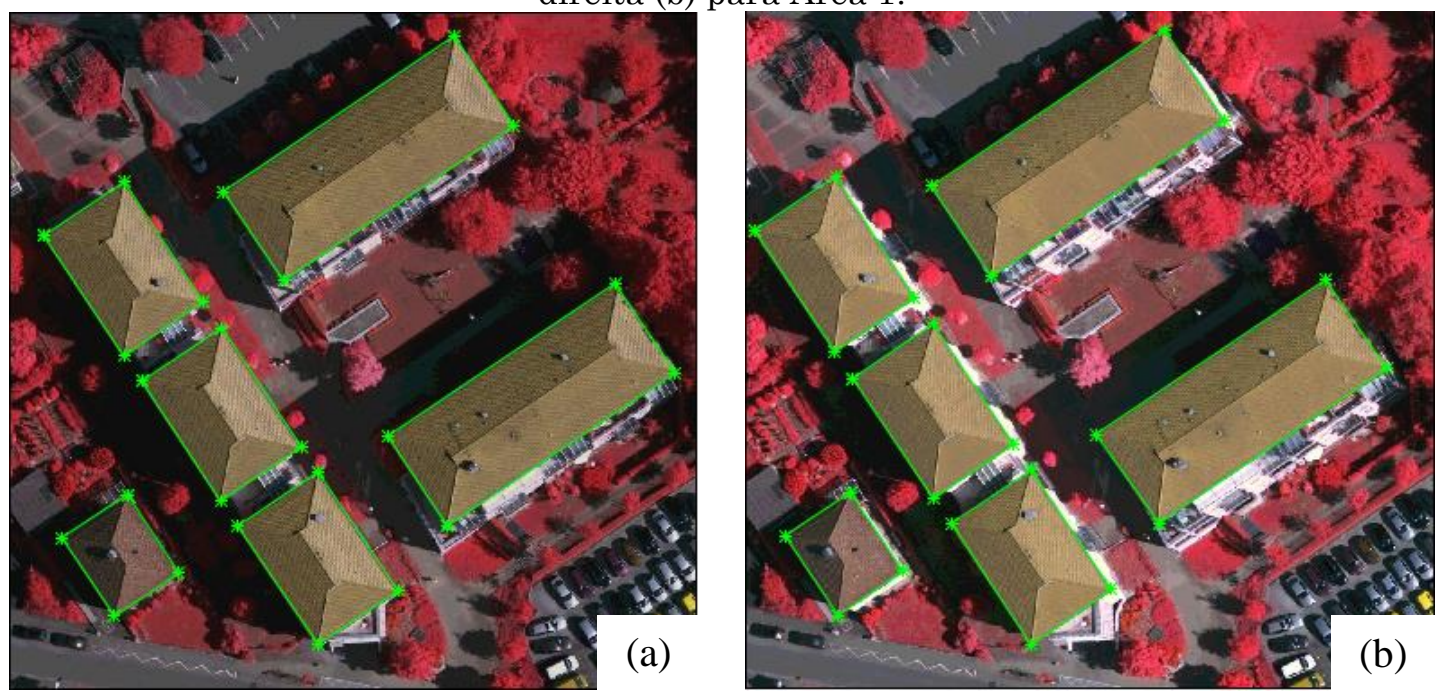

Fonte: Elaborado pelos autores

Com base na Figura 9, verificou-se que a obtenção dos cantos e retas ajustadas para cada lado do telhado das edificações mostrou-se coerente. No entanto, no caso da edificação que possui a projeção de uma sombra sobre um lado do telhado (Figura 8a), o contorno e seus respectivos cantos apresentaram um deslocamento em relação ao lado real do telhado de edificação. 
Fig. 10 - Cantos e contornos finais obtidos sobre a imagem da esquerda (a) e imagem da direita (b) para Área 2.
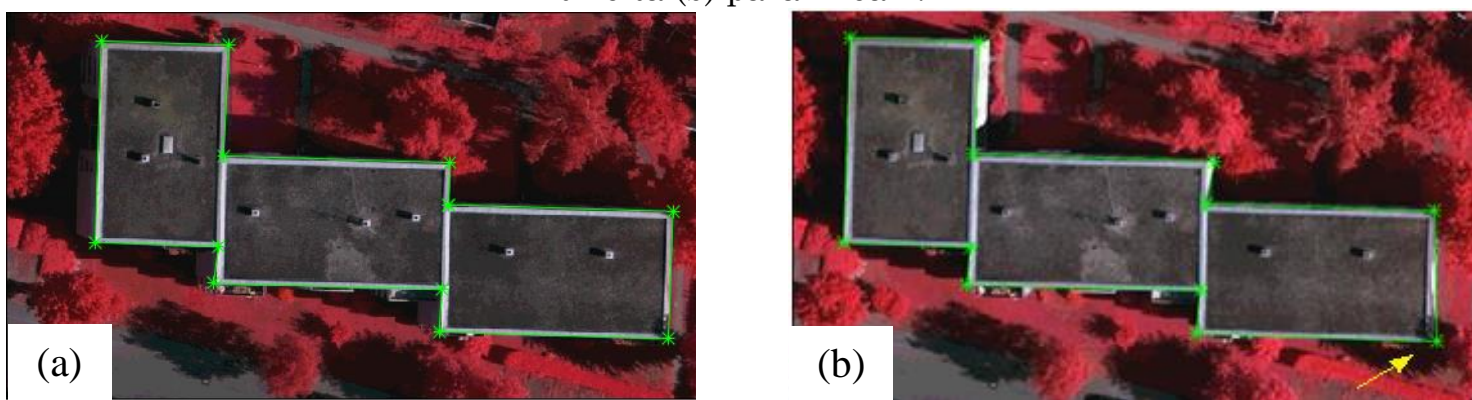

Fonte: Elaborado pelos autores

As edificações da Área 2, consideradas como uma única feição a partir do método de separação automática com base no crescimento de região, tiveram os seus cantos determinados de forma satisfatória. Os contornos obtidos apresentam coerência, porém os lados internos comuns às edificações conjugadas não foram determinados (Figura 10). Além disso, pôde-se verificar uma pequena sombra projetada (indicada pela seta amarela) da edificação que não influenciou na determinação do seu contorno com qualidade, tendo em vista que, neste caso, os pontos de borda pertencentes à borda da sombra possivelmente foram excluídos por meio do teste Tau e não considerados no ajuste de retas final como pode ser visto na Figura 7. Além disso, na Figura 10b, é possível verificar que a sombra da edificação projetada na parede da edificação vizinha foi determinada erroneamente como contorno da edificação.

$\mathrm{Na}$ Figura 11 são apresentados os cantos e retas ajustadas para as imagem da esquerda e direita para a Área 3.

Fig. 11 - Cantos e contornos finais obtidos sobre a imagem da esquerda (a) e imagem da direita (b) para Área 3. 

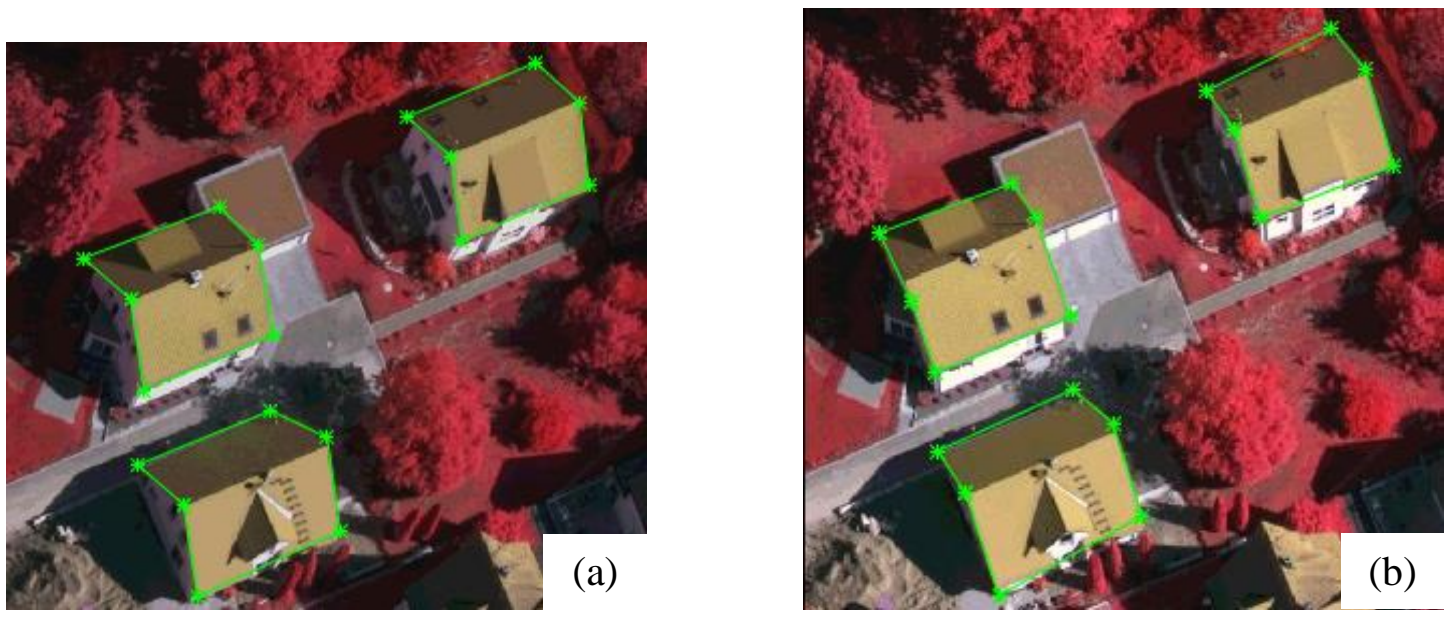

Fonte: Elaborado pelos autores.

Com base na Figura 11, verificou-se que os cantos e os contornos finais obtidos para os lados de edificações da imagem da esquerda correspondente à Área 3 foram determinados de forma condizente, a não ser por um pequeno deslocamento no contorno do lado da edificação que possui sombra de árvores projetadas, como já mencionado.

Neste trabalho, a avaliação quantitativa do método desenvolvido foi realizada por meio da comparação das coordenadas $3 \mathrm{D}$, no espaço objeto, dos cantos das edificações, obtidas a partir da intersecção fotogramétrica das coordenadas imagem determinadas pelo método proposto. As coordenadas 3D usadas como referência $\left(\mathrm{X}_{\mathrm{i}}^{\mathrm{R}}\right)$ foram obtidas a partir da intersecção fotogramétrica com base em leituras monoscópicas feitas por um operador. Deste modo, disponíveis as coordenadas de referência $\left(\mathrm{X}_{\mathrm{i}}^{\mathrm{R}}\right)$ e as coordenadas determinadas pelo método proposto $\left(\mathrm{X}_{\mathrm{i}}^{\mathrm{C}}\right)$, foi possível calcular a raiz do erro médio quadrático (REMQ) de cada componente.

As Tabelas 1 e 2 mostram a média dos valores resultantes para planimetria e altimetria, respectivamente, em cada área. Considerando que o GSD da imagem seja 0,08 m, a média é expressa também em valores de GSD.

Tabela 1 - Média dos valores de REMQ para planimetria dos telhados de edificações das áreas.

\begin{tabular}{c|c}
\hline REMQ \\
\cline { 2 - 2 }
\end{tabular}




\begin{tabular}{c|c|c}
\cline { 2 - 3 } & Horizontal $(\mathrm{m})$ & Horizontal (GSD) \\
\hline Área 1 & 0,078 & 0,97 \\
\hline Área 2 & 0,186 & 2,32 \\
\hline Área 3 & 0,218 & 2,63 \\
\hline \multicolumn{2}{|c}{ Fonte: Elaborado pelos autores }
\end{tabular}

Tabela 2 - Média dos valores de REMQ para altimetria dos telhados de edificações das áreas analisadas.

\begin{tabular}{c|c|c}
\hline \multirow{2}{*}{} & \multicolumn{2}{|c}{ REMQ } \\
\cline { 2 - 3 } & Vertical (m) & Vertical (GSD) \\
\hline Área 1 & 0,144 & 1,80 \\
\hline Área 2 & 1,054 & 13,17 \\
\hline Área 3 & 0,470 & 5,87 \\
\hline \multicolumn{2}{|c}{ Fonte: Elaborado pelos autores }
\end{tabular}

Comparando as três áreas de estudo, a Área 1, considerada como a situação ideal (desconsiderando a pequena sombra projetada em uma de suas edificações), resultou no melhor desempenho segundo os valores médios de REMQ obtidos para planimetria e altimetria, que foram aproximadamente, $8 \mathrm{~cm}$ e $14 \mathrm{~cm}$, correspondentes a 0,97 GSD e 1,80 GSD, respectivamente.

\section{Conclusões e Recomendações}

Este trabalho teve como objetivo o desenvolvimento e implementação de um método para a extração de contornos de telhados de edificações por meio da combinação de dados LiDAR e imagens aéreas digitais.

Assim como em Chang et al. (2014) e Marcato e Dal Poz (2014), neste trabalho foi utilizado o detector de Canny para a detecção de bordas nas imagens ópticas que, por sua vez, apresentou bons resultados ao considerar os parâmetros estabelecidos, possibilitando a detecção das bordas de edificações. No entanto, uma limitação nesta etapa foi a presença de sombras de vegetação, por exemplo, ou qualquer outro objeto sobre os telhados de edificações, que resultaram em bordas não condizentes aos seus contornos. Para refinar as bordas das edificações detectadas por Canny, os pontos LiDAR

Rev. Bras. de Cartografia, vol. 70, n. 4, outubro/dezembro, 2018. pp. 1378 - 1408. 
dos contornos de telhados foram projetados na imagem, visando a identificação das bordas das edificações na imagem. Este procedimento foi adotado para obter o contorno das edificações com maior exatidão, tendo em vista que nesse estudo a resolução da imagem é melhor que a do levantamento LiDAR, admitindo-se que as técnicas autônomas empregadas nesse trabalho para a obtenção dos contornos de uma edificação possuem desempenhos equivalentes.

Os resultados obtidos nos experimentos realizados mostraram que o método desenvolvido conseguiu detectar os contornos de telhados de edificações, com melhor desempenho no caso de edificações isoladas, sem interferências de sombras ou quaisquer objetos sobre os telhados.

A redução do espaço urbano para a construção de imóveis provocou uma mudança significativa na forma das edificações urbanas, sendo bastante comum a presença de edificações com diferentes níveis de telhado. Desta forma, recomenda-se, o desenvolvimento de metodologias para a extração destes tipos de edificações e também de edificações conectadas, bem como o estudo de tratamento de sombras e vegetação próximas a telhados, a fim de minimizar os efeitos causados na extração de edificações.

\section{Agradecimentos}

Os autores agradecem ao Conselho Nacional de Desenvolvimento Científico e Tecnológico ( $\mathrm{CNPq}$ ) pelo financiamento dessa pesquisa junto ao Programa de Pós-Graduação em Ciências Cartográficas, bem como o suporte ao projeto CNPq - Proc. 307788/2012-1.

\section{Referências}

ASPRS LAS Specification - Version 1.4 - R13, July, 2013. Site: www.asprs.com. Acessado em: junho de 2015. 
AWRANGJEB, M.; RAVANBAKHSH, M.; FRASER, C. S. Automatic detection of residential buildings using LiDAR data and multispectral imagery. ISPRS Journal of Photogrammetry \& Remote Sensing, v. LXV, 2010, pp. 457467.

BARBOSA, L. J.; GALO, M. Extração de edificações a partir de dados lidar e imagens obtidas por sensores ópticos. Relatório Parcial PIBIC, UNESP Universidade Estadual Paulista, FCT - Campus de Presidente Prudente. 2015.

CAMARGO, P. O. Ajustamento de Observações: notas de aula. Departamento de Cartografia. Faculdade de Ciências e Tecnologia - UNESP, Presidente Prudente, SP, 2000.

CANNY, J. A. Computational Approach to Edge Detection. IEEE Transactions on Pattern Analysis and Machine Intelligence, v. VIII, 1986, pp. 679-698.

CHANG, T.; ZHAI, R.; FU, J. Digital Building Model Generation through the Integration of Aerial LiDAR and Multiple Photo Data in Urban Areas. Proceedings of SPIE, p. 10, 2014.

CRAMER, M. The DGPF test on digital aerial camera evaluation - overview and test design. Photogrammetrie - Fernerkundung - Geoinformation (PFG), 2010, pp. 73-82.

DAL POZ, A. P. Filtragem de dados de varredura a laser: Princípios e exemplos. In: Simpósio Brasileiro de Sensoriamento Remoto (SBSR), Foz do Iguaçu, 2013.

GILANI, S. A. N.; AWRANGJEB, M.; LU, G. An automatic building extraction and regularisation technique using LiDAR point cloud data and Orthoimage. Remote Sensing, v. VIII, n. 3, 2016, p. 258.

HABIB, A. F.; GHANMA, M. S.; MORGAN, M. F.; MITISHITA, E. Integration of Laser and Photogrammetric Data for Calibration Purposes. XX ISPRS Congress. Istanbul: [s.n.]. 2004. pp. 12-23.

KABOLIZADE, M.; EBADI, K.; AHMADI, S. An improved snake model for automatic extraction of buildings from urban aerial images and LiDAR data. The International Archives of the Photogrammetry, Remote Sensing and Spatial Information Sciences, Saint - Mandé, v. XXXVIII, 2010, pp. 435441. 
KARSLI, F.; DIHKAN, M.; ACAR, H.; OZTURK, A. Automatic building extraction from very high-resolution image and LiDAR data with SVM algorithm. Arabian Journal of Geosciences, v. IX, 2016, p. 635.

KHOSHELHAM, K.; NARDINOCCHI, C.; FRONTONI, E.; MANCINI, A.; ZINGARETTI, P. Performance evaluation of automated approaches to building detection in multi-source aerial data. ISPRS Journal of Photogrammetry and Remote Sensing, v. LXV, 2010, pp. 123-133.

LIU, C.; HANGBIN, W.; YUNLING, Z. Extraction of Urban 3D features from LiDAR data fused with aerial images using an improved Mean Shift Algorithm. Survey Review, v. XLIII, 2011, pp. 402-414.

MARCATO, V. J.; DAL POZ, A. P. Refinamento geométrico de contornos e cumeeiras de telhados de edifícios extraídos de dados laser com uso de imagem aérea. Bol. Ciênc. Geod., Curitiba, v. 20, 2014, pp. 647-668.

OLIVEIRA, G. R. K. Uso integrado de dados LiDAR e imagens aéreas aplicado na extração de contornos de telhados de edificações. Dissertação (mestrado) - Universidade Estadual Paulista Júlio de Mesquita Filho (Unesp), Programa de Pós-Graduação em Ciências Cartográficas. Presidente Prudente-SP, p. 105. 2016.

POPE, A. J. The statistics of residuals and the detection of outliers. NOAA Technical Reports NOS 65 NGS1, Maryland/USA, 1976.

ROTTENSTEINER, F.; SOHN, G.; GERKE, M.; WEGNER, J.D. ISPRS Test Project on Urban Classification and 3D Building Reconstruction, 2013. Site:

$<$ http://www2.isprs.org/tl_files/isprs/wg34/docs/ComplexScenes_revision_v4.p df>. Acessado em janeiro de 2016.

YONG, L.; HUAYI, W. Adaptive building edge detection by combining LiDAR data and aerial images. International Archives of the Photogrammetry, Remote Sensing and Spatial Information Sciences, 2008, v. XXXVII, pp. 197-202.

ZHAI, R. Complex building detection through integrating LiDAR and aerial photos. The International Archives of the Photogrammetry, Remote Sensing and Spatial Information Sciences, Ávila, v. 40, 2015, pp. 25-27. 
Rev. Bras. de Cartografia, vol. 70, n. 4, outubro/dezembro, 2018. pp. 1378 - 1408. 